Mathematical Sciences And Applications E-Notes

Volume 3 No. 2 PP. 34-44 (2015) @ MSAEN

\title{
ON A $M^{[X]} / G / 1$ QUEUEING SYSTEM WITH GENERALIZED COXIAN-2 SERVICE AND OPTIONAL GENERALIZED COXIAN-2 VACATION
}

\author{
KAILASH C. MADAN
}

(Communicated by Türkan ERBAY DALKILIÇ)

\begin{abstract}
We study the steady state behaviour of a batch arrival single server queue in which the first service with general service times $G_{1}$ is compulsory and the second service with general service times $G_{2}$ is optional. We term such a two phase service as generalized Coxian-2 service. Just after completion of a service the server may take a vacation of random length of time with general vacation times $V_{1}$. After completion of the first phase of vacation the server may or may not take the second optional vacation with general vacation times $V_{2}$. We term this two phase vacation as optional generalized Coxian-2 sever vacation. We obtain steady state probability generating functions for the queue size at a random epoch of time in explicit and closed forms. Some particular cases of interest including some known results have been derived.
\end{abstract}

\section{INTRODUCTION}

Many authors including [8], [13], [5], [7], [3, 4], [6], [14], [15], [16], [17], [2], [1], $[18]$ and $[10,11,12]$ have studied queues with server vacations, assuming various vacation policies including Bernoulli schedules. In the present paper, we study steady state behaviour of an $M^{[X]} / G / 1$ queue with Bernoulli schedules and Coxian2 server vacations, using the supplementary variable technique. The mathematical model of our study is briefly described by the following underlying assumptions:

- Customers arrive at the system in batches of variable size in accordance with a compound Poisson process. Let $\lambda c_{i} d t$ (for $i=1,2,3, \ldots$ ) be the first order probability that a batch of $i$ customers arrives at the system during a short interval of time $(t, t+d t]$, where $0 \leq c_{i} \leq 1, \sum_{i=1}^{\infty} c_{i}=1$ and $\lambda>0$ is the mean arrival rate of batches. The arriving batches wait in the queue in the order of their arrival. It is further assumed that customers with each

Date: Received: October 08, 2014; Revised: June 06, 2015; Accepted: July 1, 2015.

2010 Mathematics Subject Classification. 60 K:25.

Key words and phrases. Batch arrivals, generalized Coxian-2 service, generalized Coxian-2 vacation, steady state.

This article is the written version of author's plenary talk delivered on August 25-28, 2014 at 3rd International Eurasian Conference on Mathematical Sciences and Applications IECMSA-2014 at Vienna, Austria. 
batch are pre-ordered for the purpose of service.

- There is a single server who provides generalized Coxian-2 service which means essential first phase of service followed by optional second phase of service. The first phase of service is provided to all customers one by one on a first-come, first-served basis. Let $S_{1}$ and $S_{2}$ be the service times for phase 1 and phase 2 , respectively. Let $A_{1}\left(s_{1}\right)$ and $a_{1}\left(s_{1}\right)$ respectively be the distribution function and the density function of the first phase service time and let $\mu_{1}(x) d x$ be the conditional probability of completion of first phase service, given that the elapsed time is $x$, so that

$$
\mu_{1}(x)=\frac{a_{1}(x)}{1-A_{1}(x)},
$$

and, therefore,

$$
a_{1}\left(s_{1}\right)=\mu_{1}\left(s_{1}\right) \exp \left(-\int_{0}^{s_{1}} \mu_{1}(x) d x\right) .
$$

- After completion of the first phase of service, the server provides second phase of service which is optional. A customer may take second phase of service with probability $\alpha$ or may leave the system with probability $1-\alpha$. Let $A_{2}\left(s_{2}\right)$ and $a_{2}\left(s_{2}\right)$ respectively be the distribution function and the density function of the second phase service time and let $\mu_{2}(x) d x$ be the conditional probability of completion of second phase service, given that the elapsed time is $x$, so that

$$
\mu_{2}(x)=\frac{a_{2}(x)}{1-A_{2}(x)},
$$

and, therefore,

$$
a_{2}\left(s_{2}\right)=\mu_{2}\left(s_{2}\right) \exp \left(-\int_{0}^{s_{2}} \mu_{2}(x) d x\right) .
$$

- As soon as the service of a customer is completed, then with probability $p$ the server may opt to take a vacation, or else with probability $1-p$ he may continue staying in the system. In queueing literature this phenomenon is termed as Bernoulli schedules. Most of the papers dealing with the Bernoulli schedules assume that on any service completion epoch the server may take a vacation with probability $p$ or may not take a vacation with probability $1-p$. They further assume that whenever the server becomes idle on completing a service (i.e., he servers the last customer present in the queue), he must necessarily take a vacation at such an epoch. However, in the present paper, we assume that on any service completion epoch the server may take a vacation with probability $p$ or may not take a vacation with probability $1-p$, irrespective of whether there are customers waiting in queue or not. We may term this phenomenon as uniform Bernoulli schedules.

- Whenever the server decides to take a vacation, his vacation period follows a generalized Coxian-2 distribution which implies that this period is bifurcated into two parts; phase 1 vacation followed by optional phase 2 
vacation. Let $V_{1}$ and $V_{2}$ denote the long vacation and short vacation times, respectively. Let $B_{1}\left(v_{1}\right)$ and $b_{1}\left(v_{1}\right)$ respectively be the distribution function and the density function of the first phase vacation time and let $\mathcal{V}_{1}(x) d x$ be the conditional probability of completion of first phase vacation, given that the elapsed vacation time is $x$, so that

$$
\mathcal{V}_{1}(x)=\frac{b_{1}(x)}{1-B_{1}(x)}
$$

and, therefore,

$$
b_{1}\left(v_{1}\right)=\mathcal{V}_{1}\left(v_{1}\right) \exp \left(-\int_{0}^{v_{1}} \mathcal{V}_{1}(x) d x\right) .
$$

- After completion of the first phase of vacation, the server may take second phase of vacation with probability $\beta$ or may return to the system with probability $1-\beta$. Let $B_{2}\left(v_{2}\right)$ and $b_{2}\left(v_{2}\right)$ respectively be the distribution function and the density function of the second phase vacation time and let $\mathcal{V}_{2}(x) d x$ be the conditional probability of completion of second phase vacation, given that the elapsed time is $x$, so that

$$
\mathcal{V}_{2}(x)=\frac{b_{2}(x)}{1-B_{2}(x)},
$$

and, therefore,

$$
b_{2}\left(v_{2}\right)=\mathcal{V}_{2}\left(v_{2}\right) \exp \left(-\int_{0}^{v_{2}} \mathcal{V}_{2}(x) d x\right) .
$$

- On completion of a vacation the server instantly takes up a customer (at the head of the queue) for service if there are customers waiting in the queue. However, if on returning back the server finds the queue empty, the server remains idle until a new customer arrives in the system.

- Various stochastic processes involved in the system are independent of each other.

\section{Definitions And Notations}

We assume that $W_{n}^{(j)}(x, t), j=1,2$, is the probability that at time $t$, there are $n(\geq 0)$ customers in the queue excluding one customer in $j$-th phase service with elapsed service time $x$. Accordingly, $W_{n}^{(j)}(t)=\int_{x=0}^{\infty} W_{n}^{(j)}(x, t) d x$ denotes the probability that at time $t$, there are $n$ customers in the queue excluding one customer in the $j$-th phase service irrespective of the value of $x$. Next, we define $V_{n}^{(j)}(x, t), j=1,2$, to be the probability that at time $t$, there are $n(\geq 0)$ customers in the queue and the server is on ,-th phase vacation with elapsed vacation time $x$. Accordingly, $V_{n}^{(j)}(t)=\int_{x=0}^{\infty} V_{n}^{(j)}(x, t) d x$ denotes the probability that at time $t$, there are $n$ customers in the queue and the server is on $j$-th phase vacation irrespective of the value of $x$. Further, let $P_{n}(t)=\sum_{j=1}^{2} W_{n}^{(j)}(t)+\sum_{j=1}^{2} V_{n}^{(j)}(t)$ denote the probability that at time $t$ there are $n(\geq 0)$ customers in the queue irrespective of 
whether the server is providing service or is on vacation. Finally, let $Q(t)$ be the probability that at time $t$, there is no customer in the system and the server is idle.

Further, for $j=1,2$, let the following denote the corresponding steady state probabilities:

$$
\begin{aligned}
& \lim _{t \rightarrow \infty} W_{n}^{(j)}(x, t)=W_{n}^{(j)}(x), \lim _{t \rightarrow \infty} W_{n}^{(j)}(t)=W_{n}^{(j)}, \\
& \lim _{t \rightarrow \infty} V_{n}^{(j)}(x, t)=V_{n}^{(j)}(x), \lim _{t \rightarrow \infty} V_{n}^{(k)}(t)=V_{n}^{(k)}, \\
& \lim _{t \rightarrow \infty} P_{n}(t)=\sum_{j=1}^{2} \lim _{t \rightarrow \infty} W_{n}^{(j)}(t)+\sum_{j=1}^{2} \lim _{t \rightarrow \infty} V_{n}^{(j)}(t)=P_{n} \\
& \lim _{t \rightarrow \infty} Q(t)=Q .
\end{aligned}
$$

Next, we define the following probability generating functions (pgf's) for $|z| \leq 1$ and $j=1,2$ :

$$
\begin{aligned}
W^{(j)}(x, z) & =\sum_{n=0}^{\infty} z^{n} W_{n}^{(j)}(x), W^{(j)}(z)=\sum_{n=0}^{\infty} z^{n} W_{n}^{(j)}, \\
V^{(j)}(x, z) & =\sum_{n=0}^{\infty} z^{n} V_{n}^{(j)}(x), V^{(j)}(z)=\sum_{n=0}^{\infty} z^{n} V_{n}^{(j)}, \\
P(z) & =\sum_{n=0}^{\infty} z^{n} P_{n}=\sum_{n=0}^{\infty} z^{n}\left(\sum_{j=1}^{2} W_{n}^{(j)}+\sum_{j=1}^{2} V_{n}^{(j)}\right), \\
C(z) & =\sum_{i=1}^{\infty} z^{i} c_{i} .
\end{aligned}
$$

Further, we define the Laplace-Steiltjes transform of the $j$-th phase service time:

$$
\bar{A}^{(j)}[\lambda-\lambda C(z)]=\int_{0}^{\infty} e^{[\lambda-\lambda C(z)] x} d A^{(j)}(x), j=1,2,
$$

and the Laplace-Steiltjes transform of the $k$-th phase vacation time:

$$
\bar{B}^{(j)}[\lambda-\lambda C(z)]=\int_{0}^{\infty} e^{[\lambda-\lambda C(z)] x} d B^{(j)}(x), j=1,2 .
$$

\section{Steady State Equations Governing the System}

The usual probability arguments lead to the following steady state equations.

$$
\begin{gathered}
\frac{d}{d x} W_{n}^{(1)}(x)+\left(\lambda+\mu_{1}(x)\right) W_{n}^{(1)}(x)=\lambda \sum_{i=1}^{n} c_{i} W_{n-i}^{(1)}(x), n \geq 1, \\
\frac{d}{d x} W_{0}^{(1)}(x)+\left(\lambda+\mu_{1}(x)\right) W_{0}^{(1)}(x)=0, \\
\frac{d}{d x} W_{n}^{(2)}(x)+\left(\lambda+\mu_{2}(x)\right) W_{n}^{(2)}(x)=\lambda \sum_{i=1}^{n} c_{i} W_{n-i}^{(2)}(x), n \geq 1, \\
\frac{d}{d x} W_{0}^{(2)}(x)+\left(\lambda+\mu_{2}(x)\right) W_{0}^{(2)}(x)=0,
\end{gathered}
$$




$$
\begin{gathered}
\frac{d}{d x} V_{n}^{(1)}(x)+\left(\lambda+\mathcal{V}_{1}(x)\right) V_{n}^{(1)}(x)=\lambda \sum_{i=1}^{n} c_{i} V_{n-i}^{(1)}(x), n \geq 1, \\
\frac{d}{d x} V_{0}^{(1)}(x)+\left(\lambda+\mathcal{V}_{1}(x)\right) V_{0}^{(1)}(x)=0, \\
\frac{d}{d x} V_{n}^{(2)}(x)+\left(\lambda+\mathcal{V}_{2}(x)\right) V_{n}^{(2)}(x)=\lambda \sum_{i=1}^{n} c_{i} V_{n-i}^{(2)}(x), n \geq 1, \\
\frac{d}{d x} V_{0}^{(2)}(x)+\left(\lambda+\mathcal{V}_{2}(x)\right) V_{0}^{(2)}(x)=0, \\
\lambda Q=(1-\beta) \int_{0}^{\infty} V_{0}^{(1)}(x) \mathcal{V}_{1}(x) d x+\int_{0}^{\infty} V_{0}^{(2)}(x) \mathcal{V}_{2}(x) d x \\
+(1-p)(1-\alpha) \int_{0}^{\infty} W_{0}^{(1)}(x) \mu_{1}(x) d x+(1-p) \int_{0}^{\infty} W_{0}^{(2)}(x) \mu_{2}(x) d x .
\end{gathered}
$$

Equations (3-1) through (3.8) are to be solved subject to the following boundary conditions, where $n \geq 0$ :

$$
\begin{gathered}
W_{n}^{(1)}(0)=(1-p)(1-\alpha) \int_{0}^{\infty} W_{n+1}^{(1)}(x) \mu_{1}(x) d x \\
+(1-p) \int_{0}^{\infty} W_{n+1}^{(2)}(x) \mu_{2}(x) d x+(1-\beta) \int_{0}^{\infty} V_{n+1}^{(1)}(x) \mathcal{V}_{1}(x) d x \\
+\int_{0}^{\infty} V_{n+1}^{(2)}(x) \mathcal{V}_{2}(x) d x+\lambda c_{n+1} Q \\
W_{n}^{(2)}(0)=\alpha \int_{0}^{\infty} W_{n}^{(1)}(x) \mu_{1}(x) d x \\
V_{n}^{(1)}(0)=p(1-\alpha) \int_{0}^{\infty} W_{n}^{(1)}(x) \mu_{1}(x) d x+p \int_{0}^{\infty} W_{n}^{(2)}(x) \mu_{2}(x) d x \\
V_{n}^{(2)}(0)=\beta \int_{0}^{\infty} V_{n}^{(1)}(x) \mathcal{V}_{1}(x) d x
\end{gathered}
$$

4. Steady State Solution: Queue Size at a Random Epoch

Theorem 4.1. Under the model assumptions described above, the steady state probability generating function of the queue size at a random epoch is given by

$$
P(z)=\frac{\left[\begin{array}{c}
\left(\bar{A}^{(1)}[\lambda-\lambda C(z)]-1\right)+\alpha\left(\bar{A}^{(2)}[\lambda-\lambda C(z)]-1\right) \\
+p\left(\bar{B}^{(1)}[\lambda-\lambda C(z)]-1\right)+p \beta\left(\bar{B}^{(2)}[\lambda-\lambda C(z)]-1\right)
\end{array}\right] Q}{H(z)}
$$


Where

$$
\begin{aligned}
& H(z)=z-(1-p)(1-\alpha) \bar{A}^{(1)}[\lambda-\lambda C(z)] \\
& -(1-p) \alpha \bar{A}^{(1)}[\lambda-\lambda C(z)] \bar{A}^{(2)}[\lambda-\lambda C(z)] \\
& -(1-\beta) p(1-\alpha) \bar{A}^{(1)}[\lambda-\lambda C(z)] \bar{B}^{(1)}[\lambda-\lambda C(z)] \\
& -(1-\beta) p \alpha \bar{A}^{(1)}[\lambda-\lambda C(z)] \bar{A}^{(2)}[\lambda-\lambda C(z)] \bar{B}^{(1)}[\lambda-\lambda C(z)] \\
& -\beta p(1-\alpha) \bar{A}^{(1)}[\lambda-\lambda C(z)] \bar{B}^{(1)}[\lambda-\lambda C(z)] \bar{B}^{(2)}[\lambda-\lambda C(z)] \\
& -\beta p \alpha \bar{A}^{(1)}[\lambda-\lambda C(z)] \bar{A}^{(2)}[\lambda-\lambda C(z)] \bar{B}^{(1)}[\lambda-\lambda C(z)] \bar{B}^{(2)}[\lambda-\lambda C(z)], \\
& \quad Q=1-\lambda E(I)\left(E\left(S_{1}\right)+\alpha E\left(S_{2}\right)+p E\left(V_{1}\right)+p \beta E\left(V_{2}\right)\right),
\end{aligned}
$$

$E(I)$ is the mean size of the arriving batch, $E\left(S_{1}\right)$ is the mean service time of the first phase service, $E\left(S_{2}\right)$ is the mean service time of the second phase service, $E\left(V_{1}\right)$ is the mean vacation time of the first phase vacation and $E\left(V_{2}\right)$ is the mean vacation time of the second phase vacation.

Proof. Multiplying equation (3.1) by $z^{n}$, summing over $n$ and adding the result to (3.2) and using (2.1) we get

$$
\frac{d}{d x} W^{(1)}(x, z)+\left(\lambda+\mu_{1}(x)-\lambda C(z)\right) W^{(1)}(x, z)=0 .
$$

Similar operation on equations (3.3) and (3.4), (3.5) and (3.6), and (3.7) and (3.8) yield

$$
\begin{gathered}
\frac{d}{d x} W^{(2)}(x, z)+\left(\lambda+\mu_{2}(x)-\lambda C(z)\right) W^{(2)}(x, z)=0 \\
\frac{d}{d x} V^{(1)}(x, z)+\left(\lambda+\mathcal{V}_{1}(x)-\lambda C(z)\right) V^{(1)}(x, z)=0 \\
\frac{d}{d x} V^{(2)}(x, z)+\left(\lambda+\mathcal{V}_{2}(x)-\lambda C(z)\right) V^{(2)}(x, z)=0
\end{gathered}
$$

Next, we perform the similar operations on the boundary conditions (3.10), (3.11), (3.12), (3.13) and make use of equation (3.9). Thus we get

$$
\begin{gathered}
z W^{(1)}(0, z)=(1-p)(1-\alpha) \int_{0}^{\infty} W^{(1)}(x, z) \mu_{1}(x) d x \\
+(1-p) \int_{0}^{\infty} W^{(2)}(x, z) \mu_{2}(x) d x+(1-\beta) \int_{0}^{\infty} V^{(1)}(x, z) \mathcal{V}_{1}(x) d x \\
+\int_{0}^{\infty} V^{(2)}(x, z) \mathcal{V}_{2}(x) d x+(\lambda C(z)-\lambda) Q \\
W^{(2)}(0, z)=\alpha \int_{0}^{\infty} W^{(1)}(x, z) \mu_{1}(x) d x \\
V^{(1)}(0, z)=p(1-\alpha) \int_{0}^{\infty} W^{(1)}(x, z) \mu_{1}(x) d x+p \int_{0}^{\infty} W^{(2)}(x, z) \mu_{2}(x) d x, \\
V^{(2)}(0, z)=\beta \int_{0}^{\infty} V^{(1)}(x, z) \mathcal{V}_{1}(x) d x
\end{gathered}
$$


Now we integrate equations (4.4) to (4.7) between the limits 0 and $x$ and obtain

$$
\begin{aligned}
& W^{(1)}(x, z)=W^{(1)}(0, z) \exp \left(-(\lambda-\lambda C(z)) x-\int_{0}^{x} \mu_{1}(t) d t\right), \\
& W^{(2)}(x, z)=W^{(2)}(0, z) \exp \left(-(\lambda-\lambda C(z)) x-\int_{0}^{x} \mu_{2}(t) d t\right), \\
& V^{(1)}(x, z)=V^{(1)}(0, z) \exp \left(-(\lambda-\lambda C(z)) x-\int_{0}^{x} \mathcal{V}_{1}(t) d t\right), \\
& V^{(2)}(x, z)=V^{(2)}(0, z) \exp \left(-(\lambda-\lambda C(z)) x-\int_{0}^{x} \mathcal{V}_{2}(t) d t\right),
\end{aligned}
$$

where $W^{(1)}(0, z), W^{(2)}(0, z), V^{(1)}(0, z)$ and $V^{(2)}(0, z)$ are given above in equations (4.8), (4.9), (4.10) and (4.11) respectively.

Next we again integrate equations (4.12) to (4.15) w.r.t. $x$ by parts and obtain

$$
\begin{aligned}
& W^{(1)}(z)=W^{(1)}(0, z)\left(\frac{1-\bar{A}^{(1)}[\lambda-\lambda C(z)]}{\lambda-\lambda C(z)}\right), \\
& W^{(2)}(z)=W^{(2)}(0, z)\left(\frac{1-\bar{A}^{(21)}[\lambda-\lambda C(z)]}{\lambda-\lambda C(z)}\right), \\
& V^{(1)}(z)=V^{(1)}(0, z)\left(\frac{1-\bar{B}^{(1)}[\lambda-\lambda C(z)]}{\lambda-\lambda C(z)}\right), \\
& V^{(2)}(z)=V^{(2)}(0, z)\left(\frac{1-\bar{B}^{(21)}[\lambda-\lambda C(z)]}{\lambda-\lambda C(z)}\right) .
\end{aligned}
$$

Now we shall determine the integrals $\int_{0}^{\infty} W^{(1)}(x, z) \mu_{1}(x) d x, \int_{0}^{\infty} W^{(2)}(x, z) \mu_{2}(x) d x$, $\int_{0}^{\infty} V^{(1)}(x, z) \mathcal{V}_{1}(x) d x$ and $\int_{0}^{\infty} V^{(2)}(x, z) \mathcal{V}_{2}(x) d x$ appearing in the right sides of equations (4.8) to (4.11). For this purpose we multiply equations (4.4) to (4.7) by $\mu_{1}(x), \mu_{2}(x), \mathcal{V}_{1}(x)$ and $\mathcal{V}_{2}(x)$ respectively and integrate each w.r.t. $x$. Thus we obtain

$$
\begin{aligned}
& \int_{0}^{\infty} W^{(1)}(x, z) \mu_{1}(x) d x=W^{(1)}(0, z) \bar{A}^{(1)}[\lambda-\lambda C(z)], \\
& \int_{0}^{\infty} W^{(2)}(x, z) \mu_{2}(x) d x=W^{(2)}(0, z) \bar{A}^{(2)}[\lambda-\lambda C(z)], \\
& \int_{0}^{\infty} V^{(1)}(x, z) \mathcal{V}_{1}(x) d x=V^{(1)}(0, z) \bar{B}^{(1)}[\lambda-\lambda C(z)], \\
& \int_{0}^{\infty} V^{(2)}(x, z) \mathcal{V}_{2}(x) d x=V^{(2)}(0, z) \bar{B}^{(2)}[\lambda-\lambda C(z)] .
\end{aligned}
$$

Utilizing equations (4.20) to (4.23) into equations (4.8) to (4.11) we get on simplifying

$$
W^{(1)}(z)=\frac{\bar{A}^{(1)}[\lambda-\lambda C(z)]-1}{H(z)},
$$


ON A $M^{[X]} / G / 1$ QUEUEING SYSTEM WITH GENERALIZED COXIAN-2 SERVICE...

$$
\begin{gathered}
W^{(2)}(z)=\frac{\bar{A}^{(2)}[\lambda-\lambda C(z)]-1}{H(z)}, \\
V^{(1)}(z)=p \frac{\bar{B}^{(1)}[\lambda-\lambda C(z)]-1}{H(z)}, \\
V^{(2)}(z)=p \beta \frac{\bar{B}^{(2)}[\lambda-\lambda C(z)]-1}{H(z)} .
\end{gathered}
$$

Further, adding equations (4.24), (4.25), (4.26) and (4.27), we get

$$
P(z)=\frac{\left[\begin{array}{c}
\left(\bar{A}^{(1)}[\lambda-\lambda C(z)]-1\right)+\alpha\left(\bar{A}^{(2)}[\lambda-\lambda C(z)]-1\right) \\
+p\left(\bar{B}^{(1)}[\lambda-\lambda C(z)]-1\right)+p \beta\left(\bar{B}^{(2)}[\lambda-\lambda C(z)]-1\right)
\end{array}\right] Q}{H(z)},
$$

where $H(z)$ is given by (4.2).

Now in order to determine the only unknown constant Q, we employ the normalizing condition

$$
Q+P(1)=1 .
$$

We note that each factor in the right side of each of the equations (4.24) to (4.27) is indeterminate of the zero by zero form at $z=1$. Therefor, employing L'Hopital's rule, we obtain

$$
W^{(1)}(1)=\lim _{z \rightarrow 1} W^{(1)}(z)=\lim _{z \rightarrow 1}\left(\frac{\bar{A}^{(1)}[\lambda-\lambda C(z)]-1}{H(z)}\right) Q=\frac{\lambda E(I) E\left(S_{1}\right) Q}{H(z)},
$$

which is further simplified as

$$
W^{(1)}(z)=\frac{\lambda E(I) E\left(S_{1}\right) Q}{1-\lambda E(I)\left(E\left(S_{1}\right)+\alpha E\left(S_{2}\right)+p E\left(V_{1}\right)+p \beta E\left(V_{2}\right)\right)} .
$$

Similarly equations (4.25) to (4.27) yield

$$
\begin{aligned}
W^{(2)}(1) & =\lim _{z \rightarrow 1} W^{(2)}(z)=\lim _{z \rightarrow 1} \alpha\left(\frac{\bar{A}^{(2)}[\lambda-\lambda C(z)]-1}{H(z)}\right) Q \\
& =\frac{\lambda \alpha E(I) E\left(S_{2}\right) Q}{1-\lambda E(I)\left(E\left(S_{1}\right)+\alpha E\left(S_{2}\right)+p E\left(V_{1}\right)+p \beta E\left(V_{2}\right)\right)}, \\
V^{(1)}(1)= & \lim _{z \rightarrow 1} V^{(1)}(z)=\lim _{z \rightarrow 1} p\left(\frac{\bar{B}^{(1)}[\lambda-\lambda C(z)]-1}{H(z)}\right) Q \\
& =\frac{\lambda p E(I) E\left(V_{1}\right) Q}{1-\lambda E(I)\left(E\left(S_{1}\right)+\alpha E\left(S_{2}\right)+p E\left(V_{1}\right)+p \beta E\left(V_{2}\right)\right)}, \\
V^{(2)}(1)= & \lim _{z \rightarrow 1} V^{(2)}(z)=\lim _{z \rightarrow 1} p \beta\left(\frac{\bar{B}^{(2)}[\lambda-\lambda C(z)]-1}{H(z)}\right) Q \\
& =\frac{\lambda p \beta E(I) E\left(V_{2}\right) Q}{1-\lambda E(I)\left(E\left(S_{1}\right)+\alpha E\left(S_{2}\right)+p E\left(V_{1}\right)+p \beta E\left(V_{2}\right)\right)}, \\
P(1)= & \frac{\lambda E(I) Q\left(E\left(S_{1}\right)+\alpha E\left(S_{2}\right)+p E\left(V_{1}\right)+p \beta E\left(V_{2}\right)\right)}{1-\lambda E(I)\left(E\left(S_{1}\right)+\alpha E\left(S_{2}\right)+p E\left(V_{1}\right)+p \beta E\left(V_{2}\right)\right)} .
\end{aligned}
$$


Using (4.34) in the normalising equation (4.29), we obtain

$$
Q=1-\lambda E(I)\left(E\left(S_{1}\right)+\alpha E\left(S_{2}\right)+p E\left(V_{1}\right)+p \beta E\left(V_{2}\right)\right) .
$$

Further, we obtain the utilization factor of the system as

$$
\rho=1-Q=\lambda E(I)\left(E\left(S_{1}\right)+\alpha E\left(S_{2}\right)+p E\left(V_{1}\right)+p \beta E\left(V_{2}\right)\right) .
$$

\section{Steady State Solution: Mean Queue Size at a Random Epoch}

Theorem 5.1. Under the model assumptions described above, the steady state mean queue size at a random epoch is given by

$$
\begin{aligned}
L_{q}=\lim _{z \rightarrow 1}\left(\frac{J_{1}+\alpha J_{2}+p K_{1}+p \beta K_{2}}{(1-M)^{2}}\right. & \\
& \left.+\frac{M\left[\alpha J_{1} J_{2}+2 p\left(\alpha K_{1} J_{2}+\alpha \beta J_{2} K_{2}+\beta K_{1} K_{2}+\beta J_{1} K_{2}\right)\right]}{(1-M)^{2}}\right) .
\end{aligned}
$$

where

$$
\begin{aligned}
J_{1} & =\lambda E[I(I-1)] E\left(S_{1}\right)+(\lambda E(I))^{2} E\left(S_{1}^{2}\right), \\
J_{2} & =\lambda E[I(I-1)] E\left(S_{2}\right)+(\lambda E(I))^{2} E\left(S_{2}^{2}\right), \\
K_{1} & =\lambda E[I(I-1)] E\left(V_{1}\right)+(\lambda E(I))^{2} E\left(V_{1}^{2}\right), \\
K_{2} & =\lambda E[I(I-1)] E\left(V_{2}\right)+(\lambda E(I))^{2} E\left(V_{2}^{2}\right), \\
M & =\lambda E[I] Q\left(E\left(S_{1}\right)+\alpha E\left(S_{2}\right)+p E\left(V_{1}\right)+p \beta E\left(V_{2}\right)\right) .
\end{aligned}
$$

Proof. Let $L_{q}$ denote the steady state mean queue size at a random epoch. Then using (4.27) we get

$$
L_{q}=\left.\frac{d}{d z} P(z)\right|_{z=1}=\left.\frac{d}{d z}\left(\frac{N(z)}{H(z)}\right)\right|_{z=1}
$$

where

$$
N(z)=\left[\begin{array}{c}
\left(\bar{A}^{(1)}[\lambda-\lambda C(z)]-1\right)+\alpha\left(\bar{A}^{(2)}[\lambda-\lambda C(z)]-1\right) \\
+p\left(\bar{B}^{(1)}[\lambda-\lambda C(z)]-1\right)+p \beta\left(\bar{B}^{(2)}[\lambda-\lambda C(z)]-1\right)
\end{array}\right] Q,
$$

and $H(z)$ is given by (4.2) and Q is given by (4.3).

Since both $N(z)$ and $H(z)$ are zero at $z=1$, we employ the following formula which uses L'Hopital's rule twice:

$$
L_{q}=\lim _{z \rightarrow 1} \frac{H^{\prime}(z) N^{\prime \prime}(z)-N^{\prime}(z) H^{\prime \prime}(z)}{2\left(H^{\prime}(z)\right)^{2}},
$$

where primes and double primes in (5.4) denote the first and second derivative respectively at $z=1$. Carrying out the derivatives and simplifying a lot of cumbersome algebra we obtain the results in (5.1) and (5.2). 


\section{Particular Cases}

Case 1: Two-phase general heterogeneous service and two-phase general vacation

The results of this case can be obtained from the above main results by putting $\alpha=1$ and $\beta=1$.

Case 2: Two-phase general heterogeneous service and one phase general vacation

The results of this case can be obtained from the above main results by putting $\alpha=1$ and $\beta=0$.

Case 3: One phase general service and two-phase general vacation

The results of this case can be obtained from the above main results by putting $\alpha=0$ and $\beta=1$.

Case 4: One phase general service and one phase general vacation

The results of this case can be obtained from the above main results by putting $\alpha=0$ and $\beta=0$.

Case 5: Two-phase general heterogeneous service and no vacation

The results of this case can be obtained from the above main results by putting $\alpha=1$ and $p=0$.

Case 6: One phase general service and no vacation

The results of this case can be obtained from the above main results by putting $\alpha=0$ and $p=0$.

\section{REFERENCES}

[1] Choi, B. D. and Park, K. K., The $M / G / 1$ queue with Bernoulli schedule. Queueing Systems. 7 (1990), 219-228.

[2] Cramer, M., Stationary distributions in queueing system with vacation times and limited service. Queueing Systems. 4 (1989), no. 1, 57-78.

[3] Doshi, B. T., A note on stochastic decomposition in a $G I / G / 1$ queue with vacations or set-up times. J. Appl. Prob. 22 (1985), 419-428.

[4] Doshi, B. T., Queueing systems with vacations-a survey. Queueing Systems. 1 (1986), 29-66.

[5] Fuhrman, S. W., A note on the $M / G / 1$ queue with server vacations. Operations Research. 32 (1984), 1368-1373.

[6] Keilson, J. and Servi, L. D., Oscillating random walk models for $G 1 / G / 1$ vacation systems with Bernoulli schedules. J. App. Prob. 23 (1986), 790-802.

[7] Lee, T. T., $M / G / 1 / N$ queue with vacation and exhaustive service discipline. Operations Research. 32 (1984), 774-784

[8] Levy, Y. and Yechiali, U., An $M / M / s$ queue with servers vacations. INFOR. 14 (1976), no. 2, 153163.

[9] Medhi, J., Stochastic Processes. Wiley Eastern, 1982.

[10] Madan, Kailash C., An $M / G / 1$ Queue with optional deterministic server vacations. Metron, LVII No. 3-4 (1999), 83-95. 
[11] Madan, Kailash C., An $M / G / 1$ queue with second optional service. Queueing Systems. 34 (2000), 37-46.

[12] Madan, Kailash C., On a single server queue with two-stage heteregeneous service and deterministic server vacations. International J. of Systems Science. 32 (2001), no. 7, 837-844.

[13] Scholl, M. and Kleinrock, L., On the $M / G / 1$ queue with rest periods and certain service independent queueing disciplines. Oper. Res. 31 (1983), no. 4, 705-719.

[14] Servi, L. D., D/G/1 queue with vacation. Operations Research. 34 (1986), no. 4, 619-629.

[15] Servi, L. D., Average delay approximation of $M / G / 1$ cyclic service queue with Bernoulli schedules, IEEE J. Sel. Areas Comm. 4 (1986), no. 6, 813 - 822.

[16] Shanthikumar, J. G., On stochastic decomposition in The $M / G / 1$ type queues with generalized vacations. Operations Research. 36 (1988), 566-569.

[17] Shanthikumar, J. G. and Sumita, Ushio, Modified Lindley process with replacement: dynamic behavior, asymptotic decomposition and applications. J. Appl. Prob. 26 (1989), 552-565.

[18] Takagi, H., Queueing Analysis, Vol. 1: Vacation and Priority Systems. North-Holland, Amsterdam, 1991.

Ahlia University, PO Box 10878, GOSI Complex, Kingdom of Bahrain.

E-mail address: kcmadan@yahoo.com; kmadan@ahlia.edu.bh 